\title{
Usefulness of a New Doppler Index for Assessing Both Ventricular Functions and Pulmonary Circulation in Newborn Piglet With Hypoxic Pulmonary Hypertension
}

\author{
TAKAHIRO SUGIURA, SATOSHI SUZUKI, MOHAMED HAMED HUSSEIN, \\ TAKENORI KATO, AND HAJIME TOGARI \\ Department of Pediatrics [T.S., M.H.H., T.K., H.T.], Nagoya City University Medical School and the \\ Department of Pediatrics, Nagoya City Johoku Hospital [S.S.], Nagoya, Japan
}

\begin{abstract}
Persistent pulmonary hypertension of the newborn is a clinical syndrome associated with a variety of cardiopulmonary diseases. Serial evaluation of pulmonary circulation and cardiac function is important, but available imaging techniques have been limited. A new Doppler index combining systolic and diastolic time intervals (the Tei index, which is a simple and noninvasive measurement) has been reported to be useful for the assessment of global cardiac function in adults and children. The purpose of this study was to test the effectiveness of the Tei index in prospectively assessing ventricular function and pulmonary circulation in a newborn piglet model with hypoxic pulmonary hypertension. One-day-old piglets $(1.1-1.6 \mathrm{~kg})$ were intubated and prepared for the experiments under room air and hypoxia. A complete two-dimensional Doppler echocardiographic examination was performed. Common hemodynamic variables were measured continuously throughout the study. The right ventricle (RV) Tei index under hypoxia (fraction of inspired oxygen = 0.10 ) was significantly higher than the value under air ventilation (medians, 0.38 versus $0.56 ; p<0.05$ ). Moreover, there was a significant correlation between RV Tei index and mean pulmonary artery pressure and positive linear correlation between individual changes in RV Tei index and changes in mPAP $\left(r^{2}=\right.$ $0.799, p<0.05)$. We conclude that the Tei index is useful for
\end{abstract}

\section{ABSTRACT}

assessing the function of the RV and the left ventricle and pulmonary circulation in a newborn piglet model with hypoxic pulmonary hypertension. These results suggest that the Tei index will become an objective method of assessing patients with persistent pulmonary hypertension of the newborn. (Pediatr Res 53: 927-932, 2003)

$\quad$ Abbreviations
PPHN, persistent pulmonary hypertension of the newborn
RV, right ventricle
LV, left ventricle
PAP, pulmonary artery pressure
HR, heart rate
mPAP, mean pulmonary artery pressure
SBP, systemic blood pressure
mSBP, mean systemic blood pressure
CVP, central venous pressure
TR, tricuspid regurgitation
PDA, patent ductus arteriosus
PFO, patent foramen ovale
ROC, receiver-operating characteristic
RVPEP/RVEP, right ventricular preejection period/right
ventricular ejection period

Persistent pulmonary hypertension of the newborn (PPHN) is a clinical syndrome associated with a variety of cardiopulmonary diseases. Infants with PPHN are severely hypoxemic owing to a sustained elevation in pulmonary vascular resistance [and the resulting elevated pulmonary artery pressure (PAP)], with associated extrapulmonary shunting $(1,2)$. PPHN can be associated with a myriad of clinical conditions, such as

Received June 17, 2002; accepted December 20, 2002.

Correspondence: Takahiro Sugiura, M.D., Department of Pediatrics, Nagoya City University Medical School, 1 Kawasumi, Mizuho-cho, Mizuho-ku, Nagoya 467-8601, Japan; e-mail: osugi@med.nagoya-cu.ac.jp

DOI: 10.1203/01.PDR.0000061540.81669.2A meconium aspiration, asphyxia, and heart failure. Thus, serial evaluation of pulmonary circulation and cardiac function is important to assess accurately the clinical course, the efficacy of treatment, and the prognosis. The available imaging techniques have been limited, but a new Doppler echocardiographic index combining systolic and diastolic time intervals (the Tei index) has been reported to be useful for the assessment of global function of the right ventricle (RV) and left ventricle (LV), independent of geometric assumptions, heart rate (HR), and blood pressure (3-9). The Tei index is defined as the sum of isovolumetric contraction time and isovolumetric relaxation time divided by the ejection time. Recently the Tei index has shown promise in the assessment of ventricular 
function in adults with primary pulmonary hypertension $(4,5)$ and in children with congenital heart disease (6-8). Furthermore, normal values of this index in children, neonates, and fetuses have been determined $(10,11)$. Because lung vascular tone changes rapidly in newborns with pulmonary vascular disease, serial measurements are sometimes necessary to examine the pulmonary reactivity in newborns with PPHN. The purpose of this study was to test the effectiveness of the Tei index in prospectively assessing the function of the RV and the LV and pulmonary circulation in a newborn piglet model with hypoxic pulmonary hypertension.

\section{METHODS}

The experimental protocol was approved by the Ethics Committee of Nagoya City University Medical School. Twenty-one mixed-strain newborn piglets were obtained on their first day of life from a local farmer. The average weight at the time of our study was $1.3 \mathrm{~kg}$ (range, $1.1-1.6 \mathrm{~kg}$ ). After anesthetizing by i.v. pentobarbital sodium $(20 \mathrm{mg} / \mathrm{kg})$, each newborn piglet underwent a tracheostomy and was intubated with an endotracheal tube $(4.0 \mathrm{~mm}$ internal diameter $)$ and ventilated with an infant ventilator (model IV-100; Sechrist, Anaheim, CA, U.S.A.). The inspiration/expiration pressures were initially set at $12 / 3-4 \mathrm{cmH}_{2} \mathrm{O}$ with an inspiration time of $0.5 \mathrm{~s}$, and then adjusted to maintain arterial partial pressure of carbon dioxide at $30-50 \mathrm{~mm} \mathrm{Hg}$ with air. Body temperature was monitored by a rectal probe and maintained at $38-39^{\circ} \mathrm{C}$ with a heating pad. A 4-Fr Berman angiographic catheter (American Edwards Laboratories; Irvine, CA, U.S.A.) was inserted using fluoroscopy via the right external carotid vein and placed in the main pulmonary artery to measure PAP. A 4-Fr polyvinyl catheter was inserted via the left external carotid vein into the right atrium to measure the central venous pressure (CVP). Two 4-Fr polyvinyl catheters were inserted via the umbilical arteries and advanced into the descending aorta: one was used for systemic blood pressure (SBP) measurements, and the other one was used for taking blood specimens. Arterial blood gas was analyzed with a standard analyzer (model 248; CIBA Corning, Medfield, MA, U.S.A.). PAP, SBP, and CVP were measured with a previously calibrated strain gauge (model 78801 B; Hewlett Packard, Andover, MA, U.S.A.). Anesthesia was maintained by continuous i.v. infusion of sodium pentobarbital $\left(5 \mathrm{mg} \cdot \mathrm{kg}^{-1} \cdot \mathrm{h}^{-1}\right)$. Maintenance i.v. fluid of $5 \%$ glucose was infused at $10 \mathrm{~mL} \cdot \mathrm{kg}^{-1} \cdot \mathrm{h}^{-1}$ via the umbilical vein to prevent hypoglycemia. Bicarbonate was infused $(15 \mathrm{mEq} / 100 \mathrm{~mL}$ i.v. fluid) to prevent severe acidosis during the periods of hypoxia. A MacLab/8s system (ADI Instruments, Mountain View, CA, U.S.A.) was used for data collection.

Echocardiographic examination and Doppler measurements. Doppler echocardiographic studies were performed using a Hewlett Packard SONOS 500 with 5.0 MHz transducers. The Doppler sample volume was set to an axial length of $2 \mathrm{~mm}$ with a wall filter setting of $200-400 \mathrm{~Hz}$. The mitral or tricuspid inflow pattern was measured from the apical four-chamber or long-axis view with the pulsed-wave Doppler sample volume positioned at the tip of the mitral or tricuspid leaflets during diastole. The LV and RV outflow velocity patterns were measured from the apical long-axis view or the parasternal shortaxis view with the pulsed-wave Doppler sample volume positioned just below the aortic or pulmonary valve. Care was taken to perform these studies with the transducer beam as close as possible to the Doppler beam, at $\leq 20^{\circ}$ in selected planes. No angle correction of the Doppler signal was made.

Doppler tracings were recorded on 0.75 -inch S-VHS videotape. Five consecutive beats were measured and averaged for each value. Doppler time intervals were measured from mitral or tricuspid inflow and LV or RV outflow Doppler tracings (Fig. 1) (3-11). The time interval $a$ is from the cessation to onset of mitral or tricuspid inflow, which is equal to the sum of isovolumetric contraction time, ejection time, and isovolumetric relaxation time. LV or RV ejection time (b) was measured as the duration of $\mathrm{LV}$ or RV outflow. The sum of isovolumetric contraction time and isovolumetric relaxation time was easily obtained by subtracting $b$ from $a$. The Tei index for the LV and $\mathrm{RV}$ was calculated as $(a-b) / b$. The intervals between onset and end of tricuspid or mitral valve inflow and ejection time were not measured on the same cardiac cycle, but all were in a regular rhythm, and there were no differences between HR on the beats selected for both types of ventricular Doppler flow measurements.

Doppler color flow imaging was used for the detection and semiquantification of tricuspid regurgitation (TR) (12), patent ductus arteriosus (PDA), and patent foramen ovale (PFO). The TR jet was observed from the apical four-chamber view or a subcostal window, PDA was imaged from the high left parasternal view, and PFO was imaged from the parasternal and subcostal window. The shunt pattern across the PDA and PFO were classified as right to left, bidirectional, left to right, or no flow detected.

Experimental protocol. After instrumentation was finalized, 17 animals were stabilized before baseline measurements. A complete two-dimensional Doppler echocardiographic examination was performed while the animal was ventilated with air (referred to here as normoxia). Thereafter hypoxia was induced

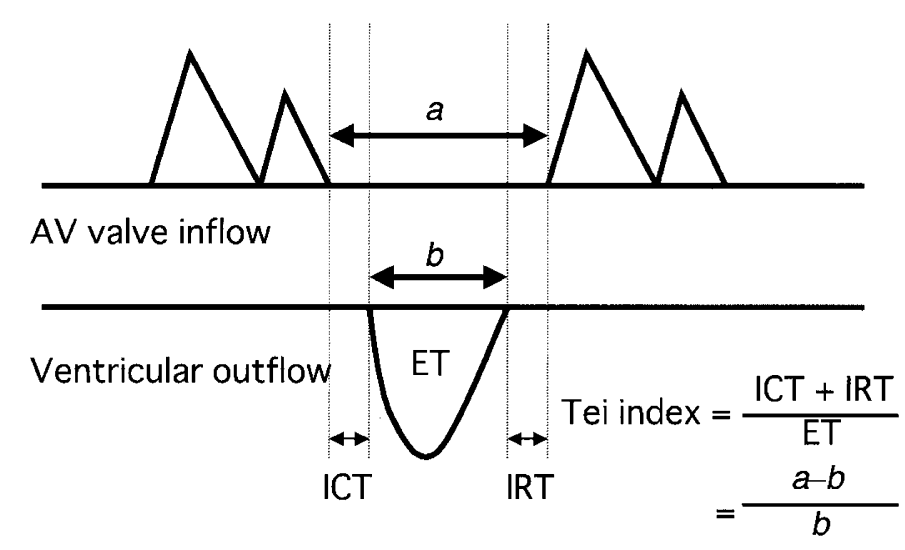

Figure 1. Measurements of Doppler time intervals. The Tei index is calculated as $(a-b) / b$. The time interval $a$ is from cessation to onset of atrioventricular (AV) valve inflow, which is equal to the sum of isovolumetric contraction time (ICT), ejection time (ET), and isovolumetric relaxation time (IRT). Ventricular ET $(b)$ was measured as the duration of left or right ventricular outflow. 
by reduction of the inspired oxygen concentration to $10 \%$ oxygen. An echocardiographic examination was then performed (referred to here as hypoxia). The experimental procedure took $<90 \mathrm{~min}$ in all cases, after which the animals were killed with a lethal dose of pentobarbital. Four control animals were studied to test whether there was a time effect on the Tei index and hemodynamic variables. After instrumentation and stabilization, echocardiographic examinations were performed at $0,30,60$, and $90 \mathrm{~min}$ in these four animals. PAP, SBP, and CVP were measured continuously throughout the control study.

Reproducibility. For evaluating the effect of observer variability on measurements of the RV and LV Tei index, five randomly selected cases (10 conditions) were analyzed by two independent observers (T.S. and S.S.) at different times, each without knowledge of the results obtained by the other.

Statistical analysis. Serial data were compared with Wilcoxon signed-rank tests. Other data were compared with the Mann-Whitney $U$ test. Significant correlations were tested with Spearman rank correlation and standard linear regression analysis. $P<0.05$ was considered significant. Except for the Tei index, data are reported as mean $\pm \mathrm{SD}$; the Tei index is reported as median and 25th and 75th percentiles. The diagnostic utility of the RV Tei index to detect hypoxia was determined using a receiver operating characteristic (ROC) curve. The results are expressed in terms of the area under the curve and the $95 \%$ confidence interval for this area. All data analyses were performed with commercially available statistical analysis software packages (Statview 5.0, Abacus Concepts, Berkeley, CA, U.S.A.; SSPS 10.0, SSPS, Chicago, IL, U.S.A.).

\section{RESULTS}

Hypoxia increased the HR, mean SBP (mSBP), and mean PAP (mPAP; HR, $184 \pm 27$ versus $224 \pm 26$ beats/min; mean $\mathrm{SBP}, 62 \pm 14$ versus $76 \pm 14 \mathrm{~mm} \mathrm{Hg}$; mPAP, $29 \pm 8.0$ versus $43 \pm 9.0$; each $p<0.05)$ but did not change the CVP $(1.7 \pm$ 1.2 versus $1.9 \pm 1.5 \mathrm{~mm} \mathrm{Hg} ; p=0.48)$. Hypoxia decreased only the arterial oxygen pressure $(78.3 \pm 17.8$ versus $21.8 \pm$ $6.5 \mathrm{~mm} \mathrm{Hg} ; p<0.05$ ), producing no changes in the $\mathrm{pH}, \mathrm{P}_{\mathrm{CO}_{2}}$, or base excess $(\mathrm{pH}, 7.39 \pm 0.07$ versus $7.36 \pm 0.07, p=0.12$; $\mathrm{PCO} 2,39.7 \pm 5.8$ versus $43.5 \pm 7.5 \mathrm{~mm} \mathrm{Hg}, p=0.12$; base excess, $-1.2 \pm 4.1$ versus $-1.8 \pm 4.2, p=0.99)$. The results of Doppler color flow imaging in hypoxia are shown in Table 1. Four (24\%) animals did not have TR jet sufficient to generate a measurable signal despite all of them having raised PAP. Only two (12\%) animals had no detectable shunt flow across the PDA. There was no detectable shunt flow through the foramen ovale in any of the animals. The RV and LV Tei indices are presented in Figure 2. The RV Tei index was higher in hypoxia than in normoxia (medians, 0.38 versus $0.56 ; p<$ 0.05 ); in contrast, the LV Tei index in hypoxia was not different from the value in normoxia (medians, 0.50 versus $0.48 ; p=0.07$ ). The sensitivity and specificity of the RV Tei index in detecting hypoxia were assessed by ROC analysis (Fig. 3). The area under the ROC curve using the RV Tei index to detect hypoxia was $0.93(0.83-1.02 ; p<0.05)$. An RV Tei
Table 1. Results of Doppler color flow imaging in hypoxia

\begin{tabular}{lc}
\hline Doppler color flow imaging & $n(\%)$ \\
\hline Tricuspid regurgitation & $3(18)$ \\
Absent or trivial & $1(6)$ \\
Mild & $12(70)$ \\
Moderate & $1(6)$ \\
Severe & \\
PDA & $0(0)$ \\
Right to left & $15(88)$ \\
Bidirectional & $0(0)$ \\
Left to right & $2(12)$ \\
No flow detected & \\
PFO & $0(0)$ \\
Right to left & $0(0)$ \\
Bidirectional & $0(0)$ \\
Left to right & $17(100)$ \\
No flow detected &
\end{tabular}
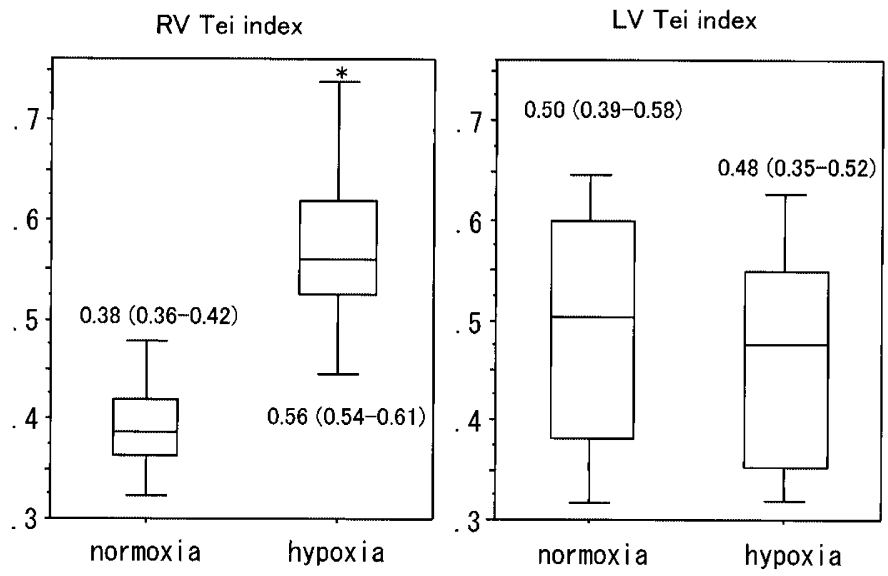

Figure 2. The values of the Tei indices in the RV and the LV in normoxia and hypoxia. Lines within the box represent the medians, boxes represent the 25 th and 75 th percentiles, and bars represent the 10th and 90th percentiles. The RV Tei index was higher in hypoxia than in normoxia (medians, 0.38 vs $0.56 ; p<$ $0.05)$.

index value of 0.43 had a sensitivity of $94 \%$ and a specificity of $88 \%$. Correlations of the RV and LV Tei index with hemodynamic parameters are shown in Table 2 . There was a correlation between the RV Tei index and mPAP i.e. Increases in the RV Tei indices were associated with the increased mPAP values (Figs. 4 and 5). There was also positive linear correlation between individual changes in $\mathrm{RV}$ Tei index (referred to as $\triangle \mathrm{RV}$ Tei index) and individual changes in mPAP (referred to as $\Delta \mathrm{mPAP} ; r^{2}=0.799, p<$ 0.05 ; Fig. 5). The RV Tei index and $\Delta \mathrm{RV}$ Tei index in animals with PDA were not different from the values in animals without PDA (median RV Tei indices, 0.39 versus $0.47, p=0.55$; median $\Delta \mathrm{RV}$ Tei indices, 0.13 versus 0.17 , $p=0.24$, respectively). In the four control animals, the Tei index and hemodynamic values remained within $10 \%$ of the initial ones. There was a positive correlation between the two independent observers' measurements of the RV and LV Tei indices (RV Tei index, $r=0.97, p<0.05$, mean absolute difference $0.03 \pm 0.02$; LV Tei index, $r=0.96, p$ $<0.05$, mean absolute difference $0.03 \pm 0.04$ ). 


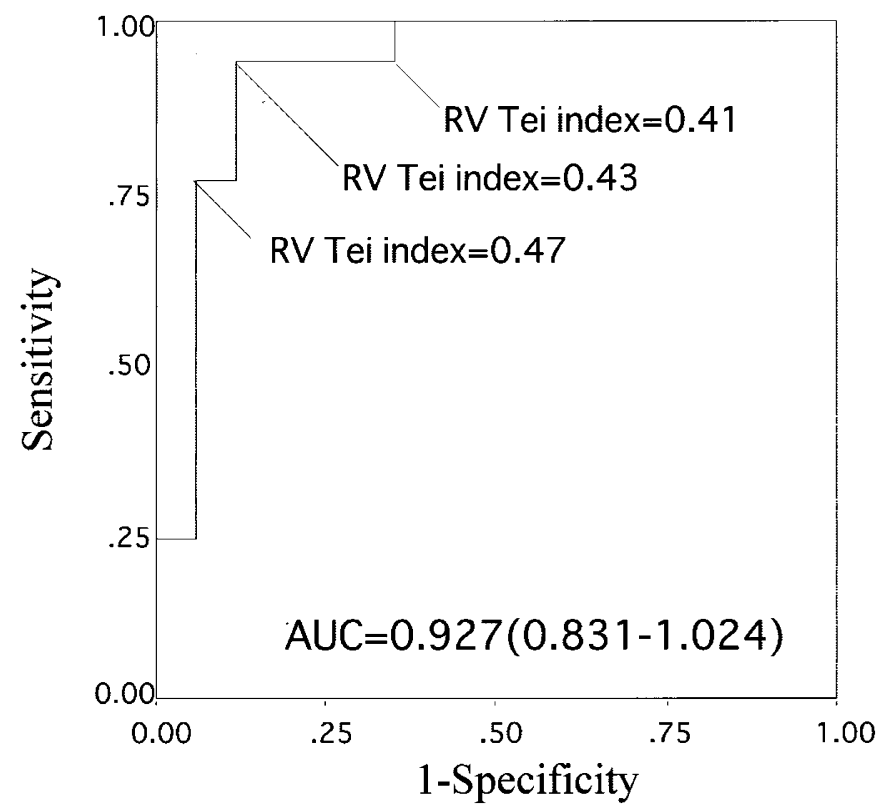

Figure 3. ROC curve examining he sensitivity and specificity of the RV Tei index to detection of hypoxia. The area under the curve was $0.927(p<0.05)$..

Table 2. Correlation of $R V$ and $L V$ Tei indices with hemodynamic parameters

\begin{tabular}{lcc}
\hline & \multicolumn{2}{c}{$p$ value } \\
\cline { 2 - 3 } & RV Tei index & LV Tei index \\
\hline HR & 0.19 & 0.25 \\
SBP & 0.38 & 0.95 \\
PAP & $<0.05$ & 0.29 \\
\hline
\end{tabular}

Probability values were obtained by Spearman rank correlation.

\section{DISCUSSION}

This study demonstrated a significantly higher RV Tei index in the hypoxic condition compared with the room-air condition, in which a positive correlation between the RV Tei index and mPAP with hypoxia and a positive correlation between $\triangle \mathrm{RV}$ Tei index and $\triangle \mathrm{mPAP}$ with hypoxia was observed. These results suggest that the Tei index will become an easy and useful quantitative parameter for assessing cardiac function and pulmonary circulation in infants with PPHN.

PPHN is a complex disorder associated with a wide array of cardiopulmonary diseases characterized by high pulmonary vascular resistance (and elevated PAP) and altered vasoreactivity, leading to right-to-left shunting of blood across the PDA and foramen ovale. Moreover, disturbances in cardiac performance compromise the tenuous balance between systemic and pulmonary circulation in $\operatorname{PPHN}(1,2)$. Thus, serial evaluation of cardiac performance is important for accurate assessment of the clinical course, the efficacy of treatment, and prognosis. For infants with PPHN, echocardiography is an ideal noninvasive method for evaluating pulmonary circulation and cardiac performance, because invasive procedures such as cardiac catheterization can affect adversely the already tenuous clinical course. To date, echocardiographic studies have been limited to the differentiation of PPHN from congenital heart disease (13), the assessment of atrial shunting (14), and the development of

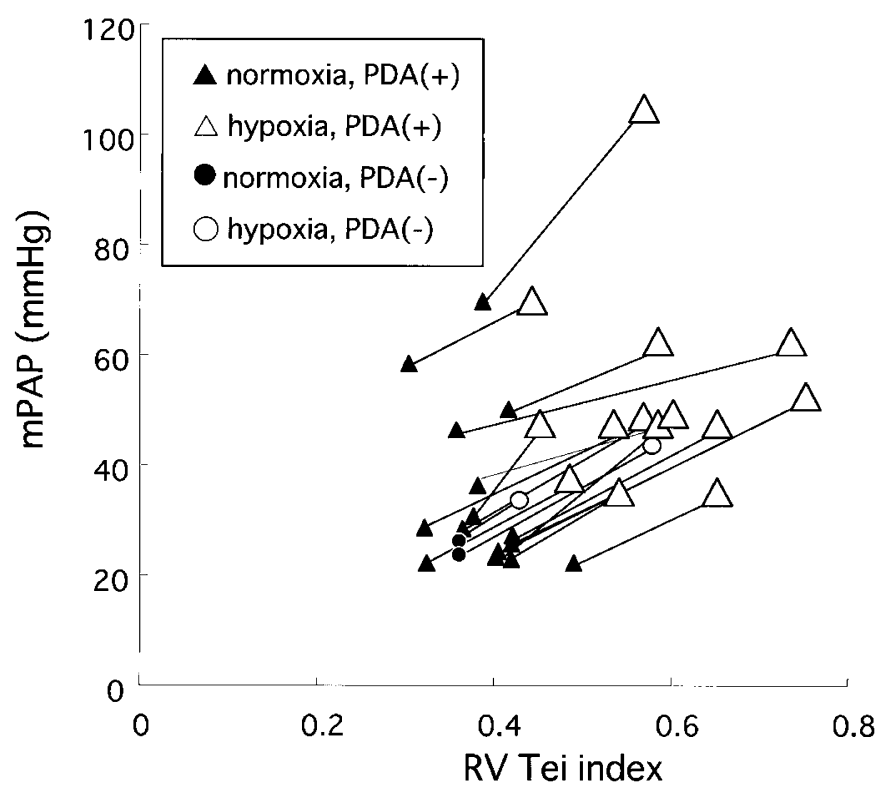

Figure 4. Serial changes of RV Tei index and mPAP. The RV Tei index increased with mPAP in all animals. The RV Tei indices in animals with PDA were not different from the values in animals without PDA (medians, $0.39 \mathrm{vs}$ $0.47 ; p=0.55)$.

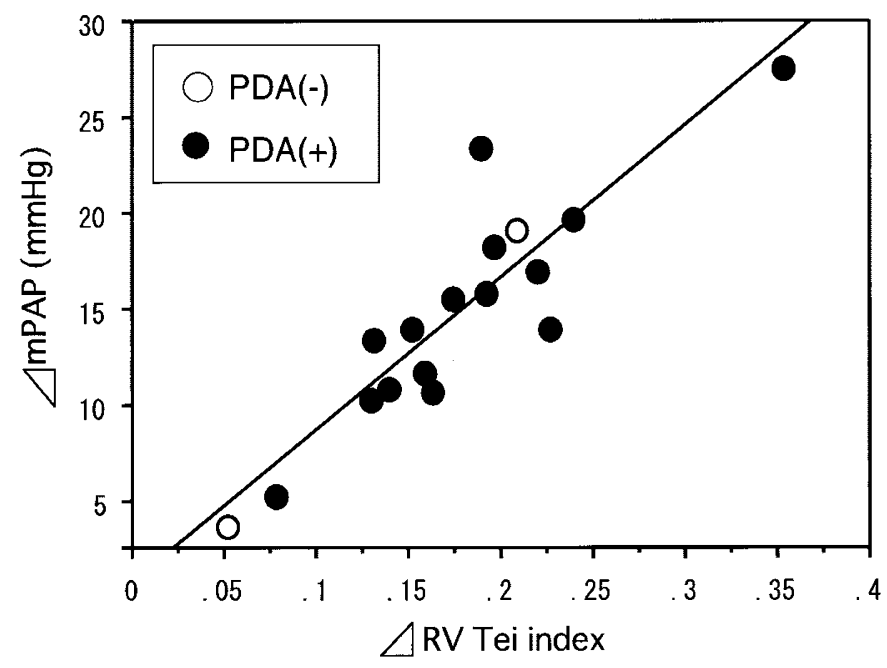

Figure 5. Correlation of individual changes in RV Tei index between normoxia and hypoxia (referred to as $\triangle \mathrm{RV}$ Tei index) with individual changes in mPAP between normoxia and hypoxia (referred to as $\triangle \mathrm{mPAP}$ ). A positive linear correlation was found between $\Delta \mathrm{RV}$ Tei index and $\triangle \mathrm{mPAP}\left(r^{2}=0.799\right.$, $p<0.05)$. $\Delta \mathrm{RV}$ Tei indices in animals with PDA were not different from the values in animals without PDA (medians, 0.13 vs $0.17 ; p=0.24$ ).

indirect M-mode indices for early identification of infants with PPHN (15). More recent Doppler methods include the use of acceleration time (16), the mean velocity of pulmonary blood flow (17), ductus venosus blood velocity (18), velocity across the PDA $(19,20)$, and regurgitant jet velocity across the tricuspid valve $(16,20,21)$. Estimation of the systolic PAP from the peak velocity of the TR jet is the most accurate method and is easy to measure $(16,21)$, but it is not always available. TR yields accurate estimates of systolic PAP when the maximal TR Doppler velocity is added to a suitable estimate of right atrial pressure $(16,21)$, but TR cannot be found in every infant with PPHN: Skinner et al. (22) reported that TR 
permitting systolic PAP estimation is not present in $30 \%$ of patients who have clinical evidence of PPHN. Similarly, PDA has been shown to yield accurate estimates of systolic PAP from noninvasive echocardiographic Doppler flow velocity measurement across it. However, it is not available when the ductus arteriosus is closed. In the present study, we demonstrate that $24 \%$ of the animals did not have sufficient TR and $12 \%$ of them did not have detectable PDA.

As mentioned at the beginning of this article, the Tei index is defined as the sum of isovolumetric contraction time and isovolumetric relaxation time divided by ejection time (3-11). Specifically, the determination of this index requires the measurement of only two simple, conventional Doppler intervals from ventricular inflow and outflow, which are available in every infant. Moreover, this index does not rely on the HR or the size, shape, or orientation of the heart (3-9); therefore, it can be applied easily in infants with PPHN.

Earlier studies suggested that similar isovolumetric index, $\mathrm{RV}$ preejection period/RV ejection period (RVPEP/RVEP), is an indirect index of PAP and useful for the diagnosis of PPHN (15). RVPEP/RVEP is obtained via M-mode echocardiographic time intervals, has a wide range of normal values in infants, and tends to correlate poorly with quantitative changes in pressure. Ochikubo et al. (20) demonstrated that presumed marked falls in the PAP of neonates with PPHN during inhaled nitric oxide therapy were not reflected by significant changes in RVPEP/RVEP. In neonatal hypoxic pulmonary hypertension, it has been suggested that RV diastolic function was more influenced than RV systolic function (23). RVPEP/RVEP should mainly reflect RV systolic function, whereas the Tei index reflects global function combining the elements of systolic and diastolic function. We did not directly compare the two indices because electrocardiograms were not simultaneously recorded with echocardiographic measurements, but our results suggest that the RV Tei index will provide results that are more accurate than those of RVPEP/RVEP.

The load dependence of the Tei index has yet to be completely evaluated, but Tei et al. (4) showed that the RV Tei index does not correlate with RV systolic pressure and diastolic pulmonary pressure in the presence of RV myocardial dysfunction. Furthermore, a recent study in congenital heart disease, which included patients with pressure load lesions and volume load lesions and others with congenitally corrected transposition of the great arteries, concluded that the Tei index is relatively independent of changes in preload or afterload in clinical settings (8). Vedrinne et al. (23) showed a positive correlation between mPAP and RV end-diastolic area in a newborn piglet model similar to ours. In our study, the RV was also dilated with hypoxia. Taken together, these results reveal a positive correlation between RV Tei index and mPAP. The RV Tei index may not be solely affected by the increased afterload - it may substantially be related to RV dilation. Eidem et al. $(6,8)$ reported that patients who had Ebstein's anomaly and a severe RV dilation demonstrated elevation of the RV Tei index.

The RV Tei index may also be affected by hypoxia, but Hillman et al. (24) demonstrated that the RV function of the animals with hypoxic pulmonary hypertension recovered after nitric oxide inhalation, which is known to have no effect on cardiac contractility and systemic circulation. This result indicates that the RV function was affected only by elevated afterload, and hypoxia had no adverse effect on RV myocardial performance. Therefore, the RV Tei index in our study would reflect the myocardial dysfunction from pure afterload elevation. In addition, we have demonstrated that PDA, which is closely related to loading conditions, had no apparent effect on the RV Tei index. Additional studies, including a large number of serial examinations, will be required to clarify the effects of loading conditions and hypoxia.

Compared with measurements in the RV, the LV Tei index in hypoxia was not different from the values in normoxia. Several studies have documented that LV afterload, which is reflected by systemic vascular resistance and LV end-systolic wall stress, decreases significantly during hypoxia $(23,25)$. However, the compression of a dilated RV with hypoxia may cause a negative effect on LV function. These factors would not affect these indices. These results suggest that there was no LV myocardial dysfunction in our cases. However, both high pulmonary vascular resistance and low systemic blood flow due to "pump failure" coexist in the same patient with PPHN. Historically, these infants may have had severe perinatal asphyxia $(26,27)$ or an obstetric complication (e.g. abruptio placentae or placenta previa) that caused significant fetal hemorrhage (28). A significant number of stressed infants experience myocardial infarcts in the context of perinatal asphyxia $(27,29)$. Poulsen et al. (9) reported that the LV Tei index was significantly higher in patients with acute myocardial infarction compared with healthy subjects. Thus, for these infants with PPHN, the LV Tei index may be useful for assessing the LV myocardial function.

\section{CONCLUSION}

The Tei index was developed for use in adult patients with pulmonary hypertension, whereas this study used the index to assess a neonatal pulmonary hypertension model. We demonstrated a significant increase in the RV Tei index and significant correlations between the RV Tei index and mPAP and between $\triangle \mathrm{RV}$ Tei index and $\triangle \mathrm{mPAP}$ in newborn piglets with hypoxic pulmonary hypertension. These results suggest that the Tei index will become an easy and useful quantitative parameter for assessing infants with PPHN.

\section{REFERENCES}

1. Steinhorn RH, Millard SL, Morin FC 1995 Persistent pulmonary hypertension of the newborn. Role of nitric oxide and endothelin in pathophysiology and treatment. Clin Perinatol 22:405-428

2. Kinsella JP, Abman SH 1995 Recent developments in the pathophysiology and treatment of persistent pulmonary hypertension of the newborn. J Pediatr 126:853864

3. Tei C, Ling LH, Hodge DO, Bailey KR, Oh JK, Rodeheffer RJ, Tajik AJ, Seward JB 1995 New index of combined systolic and diastolic myocardial performance: a simple and reproducible measure of cardiac function-a study in normals and dilated cardiomyopathy. J Cardiol 26:357-366

4. Tei C, Dujardin KS, Hodge DO, Bailey KR, McGoon MD, Tajik AJ, Seward JB 1996 Doppler echocardiographic index for assessment of global right ventricular function. J Am Soc Echocardiogr 9:838-847

5. Yeo TC, Dujardin KS, Tei C, Mahoney DW, McGoon MD, Seward JB 1998 Value of a Doppler-derived index combining systolic and diastolic time intervals in predicting outcome in primary pulmonary hypertension. Am J Cardiol 81:1157-1161 
6. Eidem BW, Tei C, O’Leary PW, Cetta F, Seward JB 1998 Nongeometric quantitative assessment of right and left ventricular function: myocardial performance index in normal children and patients with Ebstein anomaly. J Am Soc Echocardiogr 11:849856

7. Ishii M, Eto G, Tei C, Tsutsumi T, Hashino K, Sugahara Y, Himeno W, Muta H, Furui J, Akagi T, Fukiyama R, Toyoda O, Kato H 2000 Quantitation of the global right ventricular function in children with normal heart and congenital heart disease: a right ventricular myocardial performance index. Pediatr Cardiol 21:416-421

8. Eidem BW, O'Leary PW, Tei C, Seward JB 2000 Usefulness of the myocardial performance index for assessing right ventricular function in congenital heart disease. Am J Cardiol 86:654-658

9. Poulsen SH, Jensen SE, Tei C, Seward JB, Egstrup K 2000 Value of the Doppler index of myocardial performance in the early phase of acute myocardial infarction. J Am Soc Echocardiogr 13:723-730

10. Mori Y, Rice MJ, McDonald RW, Reller MD, Wanitkun S, Harada K, Sahn DJ 2001 Evaluation of systolic and diastolic ventricular performance of the right ventricle in fetuses with ductal constriction using the Doppler Tei index. Am J Cardiol 88:11731178

11. Tsutsumi T, Ishii M, Eto G, Hota M, Kato H 1999 Serial evaluation for myocardial performance in fetuses and neonates using a new Doppler index. Pediatr Int 41:722727

12. Omoto R, Yokote Y, Takamoto S, Kyo S, Ueda K, Asano H, Namekawa K, Kasai C, Kondo Y, Koyano A 1984 The development of real-time two-dimensional Dopple echocardiography and its clinical significance in acquired valvular diseases with special reference to the evaluation of valvular regurgitation. Jpn Heart J 25:325-340

13. Linday LA, Ehlers KH, O'Loughlin JE, LaGamma EF, Engle MA 1983 Noninvasive diagnosis of persistent fetal circulation versus congenital cardiovascular defects. Am J Cardiol 52:847-851

14. Sahn DJ, Allen HD, George W, Mason M, Goldberg SJ 1977 The utility of contras echocardiographic techniques in the care of critically ill infants with cardiac and pulmonary disease. Circulation 56:959-968

15. Valdes-Cruz LM, Dudell GG, Ferrara A 1981 Utility of M-mode echocardiography for early identification of infants with persistent pulmonary hypertension of the newborn. Pediatrics 68:515-525

16. Chan KL, Currie PJ, Seward JB, Hagler DJ, Mair DD, Tajik AJ 1987 Comparison of three Doppler ultrasound methods in the prediction of pulmonary artery pressure. J Am Coll Cardiol 9:549-554
17. Rozé JC, Storme L, Zupan V, Morville P, Dinh-Xuan AT, Mercier JC 1994 Echocardiac investigation of inhaled nitric oxide in the newborn babies with severe hypoxaemia. Lancet 344:303-305

18. Fugelseth D, Kiserud T, Liestøl K, Langslet A, Lindemann R 1999 Ductus venosus blood velocity in persistent pulmonary hypertension of the newborn. Arch Dis Child 81:F35-F39

19. Musewe NN, Poppe D, Smallhorn JF, Hellman J, Whyte H, Smith B 1990 Doppler echocardiographic measurement of pulmonary artery pressure from ductal Doppler Velocities in the newborn. J Am Coll Cardiol 15:446-456

20. Ochikubo CG, Waffarn F, Turbow R, Kanakriyeh M 1997 Echocardiographic evidence of improved hemodynamics during inhaled nitric oxide therapy for persistent pulmonary hypertension of the newborn. Pediatr Cardiol 18:282-287

21. Yock PG, Popp RL 1984 Noninvasive estimation of right ventricular systolic pressure by Doppler ultrasound in patients with tricuspid regurgitation. Circulation 70:657662

22. Skinner JR, Hunter S, Hey EN 1996 Haemodynamic features at presentation in persistent pulmonary hypertension of the newborn and outcome. Arch Dis Child 74:F26-F32

23. Vedrinne JM, Curtil A, Martinot S, Vedrinne C, Robin J, Franck M, Champsaur G 1998 The hemodynamic effects of hypoxemia in anesthetized pigs: a comparison between right heart catheter and echocardiography. Anesth Analg 87:21-26

24. Hillman ND, Cheifetz IM, Craig DM, Smith PK, Ungerleider RM, Meliones JN 1997 Inhaled nitric oxide, right ventricular efficiency, and pulmonary vascular mechanics: selective vasodilation of small pulmonary vessels during hypoxic pulmonary vasoconstriction. J Thorac Cardiovasc Surg 113:1006-1013

25. Coetzee A, Foex P, Holland D, Ryder A, Jones L 1984 Effect of hypoxia on the normal and ischemic myocardium. Crit Care Med 12:1027-1031

26. Reimenschneider TA, Nielsen HC, Ruttenberg HD, Jaffe RB 1976 Disturbances of the transitional circulation: spectrum of pulmonary hypertension and myocardial dysfunction. J Pediatr 89:622-625

27. Guller B, Bozic C 1972 Right-to-left shunting through a patent ductus arteriosus in a newborn with myocardial infarction. Cardiology 57:348-357

28. Paxson CL 1978 Neonatal shock in the first postnatal day. Am J Dis Child 132:509 514

29. Setzer ES, Ermocilla R, Tonkin I, John E, Sansa M, Cassady G 1980 Papillary muscle necrosis in a neonatal autopsy population: incidence and associated clinical manifestations. J Pediatr 96:289-294 